\title{
Modeling of intrinsic electron and hole trapping in crystalline and amorphous $\mathrm{ZnO}$
}

\author{
David Mora-Fonz ${ }^{* 1}$ and Alexander L. Shluger ${ }^{1}$ \\ ${ }^{1}$ Department of Physics and Astronomy, University College London, Gower Street, \\ London WC1E 6BT, United Kingdom
}

July 23, 2019

Keywords: Amorphous $\mathrm{ZnO}$, thin films, interatomic potentials, DFT, TCOs

\begin{abstract}
Recent advances in ultrafast liquid quenching and deposition of thin films on cold substrates make growing amorphous (a)-ZnO films increasingly feasible. We predict the electronic structure and electron and hole trapping properties of amorphous $\mathrm{ZnO}$ using Density Functional Theory (DFT) simulations with a hybrid density functional (h-DFT). An ensemble of fifty 324-atom structures is employed to obtain the distribution of structural and electronic properties of a-ZnO. The results demonstrate that electrons do not localize in a- $\mathrm{ZnO}$ but holes form deep localized states with average trapping energy of about $0.9 \mathrm{eV}$. We also show that dispersion at the conduction band minimum (CBM) is not affected upon amorphization, suggesting that high electron mobility should be retained. An average value of a-ZnO band gap of $3.36 \mathrm{eV}$ is calculated with no states splitting into the band gap, which accounts for no substantial detrimental effect on the optical transparency upon amorphization. These findings may have important implications for future applications of a- $\mathrm{ZnO}$ as a transparent conductor and photo-catalyst.
\end{abstract}

*david.fonz.11@ucl.ac.uk 


\section{Introduction}

Transparent conducting oxides (TCOs) combine optical transparency with high electrical conductivity. They are essential components in modern optoelectronic devices, such as liquid-crystal displays (LCDs), solar cells, and electrochromic windows. 1] Growing customer demand for flexible displays and wearable computers, ${ }^{5}$ require development of flexible TCOs. In this regard, amorphous TCOs (a-TCOs) provide several advantages over their crystalline (c) counterparts, such as flexibility, lower production cost, and smooth surfaces, while maintaining high levels of electrical conductivity and transparency. Nevertheless, some of the most studied and used aTCOs, amorphous InSnO (a-ITO) and InGaZnO (a-IGZO) include the expensive elements In and Ga. Following the a-TCO requirements, uniform and stable a-ZnO thin films would provide an excellent and much cheaper alternative for a-TCO applications.

To date, growing stable a-ZnO films remains a challenge. Samples are often (poly)crystalline, ${ }^{6}$ which affects the device performance since any repeated bending causes film fragmentation. 7 Furthermore, even thermodynamically stable phases, e.g. zincblende or rocksalt, are only grown under very specific conditions of substrates and/or at high pressure (see, for example, refs. 812). Although successful growth of thin a-ZnO films with thicknesses ranging from 5 to $100 \mathrm{~nm}$

using different techniques and on different substrates has been reported, 13 18 the morphology of these films remains controversial due to the lack of high intensity X-ray diffraction or grazing incidence X-ray and neutron diffraction characterization. Many of such films are reported to contain nanocrystalline inclusions. $\frac{13 \mid 16] 18}{20}$

Amid largely unsuccessful attempts to grow good quality a-ZnO films, theoretical predictions of a- $\mathrm{ZnO}$ have been more optimistic. Stable a- $\mathrm{ZnO}$ structures have been produced using mainly ab initio molecular dynamics (MD) melt and quench (MQ) methods. 21 26] These computationally demanding calculations use small periodic cells, very high cooling rates and provide limited statistics of structural characteristics. The sampling of the a- $\mathrm{ZnO}$ configurational space has been, therefore, poor and a distribution of electronic properties has not been provided yet.

Recently, we investigated the ability of bulk $\mathrm{ZnO}$ to form glass structures using interatomic potentials (IPs) and a MQ procedure within isothermal-isobaric (NPT) ensemble. $\stackrel{27}{\text { This allowed }}$ 
us to use large (up to 768000 atoms) periodic cells and improve the statistics of the distribution of a- $\mathrm{ZnO}$ structural characteristics. These calculations have demonstrated that cooling rates in a MQ procedure equal or exceeding $100 \mathrm{~K} \mathrm{ps}^{-1}$ lead to formation of stable and uniform amorphous structures. $\mathrm{ZnO}$ samples show, however, different degrees of crystallinity at lower cooling rates. The average density of a-ZnO samples produced using IPs is about $5.04 \mathrm{~g} \mathrm{~cm}^{-3}$ and the coordination numbers of $\mathrm{Zn}$ and $\mathrm{O}$ atoms are around 3.9, reflecting the strong propensity to crystallization. Still, the stability tests carried out using the Activation-Relaxation Technique (ART) $\underline{28[32]}$ and simulated annealing demonstrated that the obtained amorphous structures are stable. 27

Advances in ultrafast liquid quenching and deposition of thin films on cold substrates 33 36 make achieving growth of amorphous $\mathrm{ZnO}$ films a tangible prospect. Their potential use in (photo)electronic devices will expose these films to electrons and holes. Therefore, questions arise whether a-ZnO films will retain good electron mobility and whether there is any possibility for electron or hole localization due to disorder. Such localization in deep states has been predicted theoretically and supported experimentally in other amorphous oxides, such as a- $\mathrm{SiO}_{2}, \mathrm{HfO}_{2}$, $\mathrm{Al}_{2} \mathrm{O}_{3}, \mathrm{TiO}_{2}$ and others, $\stackrel{37-39}{39}$ where there is either no or very shallow polaron formation in the crystalline phase.

Localized charged traps and polarons play a crucial role in many materials chemistry applications, including photovoltaics, photochemistry, optoelectronics ${ }^{40}$ and photoconductivity. 41 In photo-catalysis, for example, electron-hole recombination is highly undesirable leading to short exciton lifetime and a poor photocatalyst. Evidence exists that holes can be trapped in polaronic states in $\mathrm{ZnO}$. Recently, the existence of photogenerated intermediate radius hole polaron in (10̄̄0) oriented ZnO single-crystal substrates has been suggested in ref. 42, Photogenerated ZnO holes are also important in hybrid systems, such as polyaniline-ZnO, where the polymer is used to enhance the oxide's photoactivity and to inhibit photocorrosion. ${ }^{43}$ Moreover, due to their photocatalycic activity, $\mathrm{ZnO}$ nanoparticles have been synthesized as anticancer and antibacterial agents. 44,48 In photochemistry, $\mathrm{ZnO}$ excitons can produce reactive oxygen species, including hydroxyl radicals $\left(\mathrm{OH}^{-}\right)$and hydrogen peroxide $\left(\mathrm{H}_{2} \mathrm{O}_{2}\right)$ from water and oxygen, which effectively decompose organic compounds. $\stackrel{49}{ }$ In the case of photoconductivity processes, it has been reported that the presence 
of hole traps in $\mathrm{ZnO}$ nanowires prevents carrier recombination, extending the photocarrier lifetime

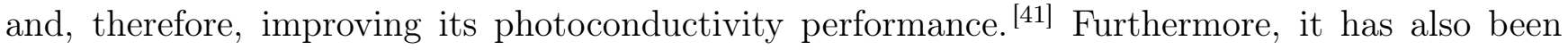
suggested that hole traps in $\mathrm{ZnO}$ may play a role in conductivity of $\mathrm{ZnO}$ varistors. $\stackrel{50}{5}$

In spite of their importance, understanding of electron and hole trapping states in a- $\mathrm{ZnO}$ is still lacking. In this work, we study the electronic structure and electron and hole trapping properties of crystalline and amorphous $\mathrm{ZnO}$ using DFT with a hybrid functional, which reproduces the experimental $\mathrm{ZnO}: \mathrm{Li}_{\mathrm{Zn}}$ defect. An ensemble of fifty 324-atom structures is employed to obtain the distribution of structural and electronic properties of a-ZnO. The results demonstrate that electrons do not localize in a- $\mathrm{ZnO}$ but holes form deep localized states with average trapping energy of about $0.9 \mathrm{eV}$-a feature not observed in c- $\mathrm{ZnO}$. Localized holes prefer to remain separated rather than forming a bipolaronic state. We also show that dispersion at the CBM is not affected upon amorphization, suggesting that high electron mobility should be retained. An average a-ZnO wide band gap of $3.36 \mathrm{eV}$ is calculated with no states splitting into the band gap, which accounts for no substantial detrimental effect on the optical transparency upon amorphization. The theoretical findings reported here may have important implications for applications of a-ZnO as a TCO and for understanding photo-induced processes in a- $\mathrm{ZnO}$.

\section{Computational Details}

Fifty a- $\mathrm{ZnO}$ structures were generated using highly accurate IPs. ${ }^{51}$. These structures are quasiperiodic with a periodic cell containing 324 atoms in a cubic 3D-periodic arrangement. Every structure was fully reoptimized, using both generalized gradient approximation (GGA) and hybrid functionals, and tested for charge localization, as discussed in more detail below.

\subsection{Amorphous Structures}

Amorphous structures were generated using an MD MQ approach with the LAMMPS code $\stackrel{52}{5}$ and a Born-Mayer-type IPs developed in ref. 51. These IPs reproduces a wide range of physical and structural properties of c- $\mathrm{ZnO}$, such as lattice parameters, thermal expansion coefficients, 
vibrational spectra, surface energies, dielectric response, elastic and piezoelectric constants, and formation energies of point defects in a good agreement with experimental data. 12[53 [55] We note that the original IPs uses a polarizable shell model, whereas in this work the computational cost was reduced by using a rigid ion model, with ionic charges of +2 and -2 on $\mathrm{Zn}$ and $\mathrm{O}$, respectively.

Every a-ZnO structure was generated, within an NPT ensemble with a Nosé-Hoover thermostat and barostat, using the following procedure: (i) equilibration of the system for 50 ps at $300 \mathrm{~K}$; (ii) temperature was linearly increased to $5000 \mathrm{~K}$ for $50 \mathrm{ps}$; (iii) the system was stabilized for 500 ps at $5000 \mathrm{~K}$; (iv) structures were cooled down to $300 \mathrm{~K}$ with a cooling rate of $100 \mathrm{~K} \mathrm{ps}^{-1}$; (v) the system was equilibrated for $50 \mathrm{ps}$ at $300 \mathrm{~K}$. Cubic periodic cells were used in all cases. Coordination numbers, radial and bond angle distribution functions were calculated with the R.I.N.G.S. code 56 using a cutoff of $2.45 \AA$. The radial cutoff was chosen at the tail of the first peak of the radial distribution function graph (see Figure 1).

Further, we have used the ART method 282$]$ to explore the energy landscape of every a- $\mathrm{ZnO}$ structure produced. A Metropolis temperature of $0.25 \mathrm{eV}$ has been used as a criterion to accept/reject new events (minima). A local distortion was used within the ART code with a cutoff radius of $3.5 \AA$. The atomic structure and stability of a- $\mathrm{ZnO}$ produced in this way are discussed in detail in ref. 27 .

\subsection{Periodic DFT calculations}

All electronic structure calculations were performed using the Gaussian and plane-wave CP2K code. ${ }^{57}$ The GGA Perdew-Burke-Ernzerhof (PBE) functional [58] was employed for initial geometry optimizations. A kinetic energy cutoff of $600 \mathrm{Ry}(8163 \mathrm{eV})$ was sufficient to converge the bulk lattice energy (four atoms unit cell) to less than $1 \mathrm{meV}$. The DZVP-MOLOPT-SR-GTH basis sets ${ }^{[59}$ were employed for all atomic species with the GTH pseudopotentials. 60 When an atomic relaxation was performed, forces on all ions were converged to less than $0.02 \mathrm{eV}^{-1}$. A 300-atom unit cell was used for the crystalline phase.

For a more accurate description of electronic structure, the hybrid PBE0-TC-LRC functional 61] with an exchange cutoff radius $(\mathrm{R})$ of $3.0 \AA$ was employed (this method is called h-DFT in further 


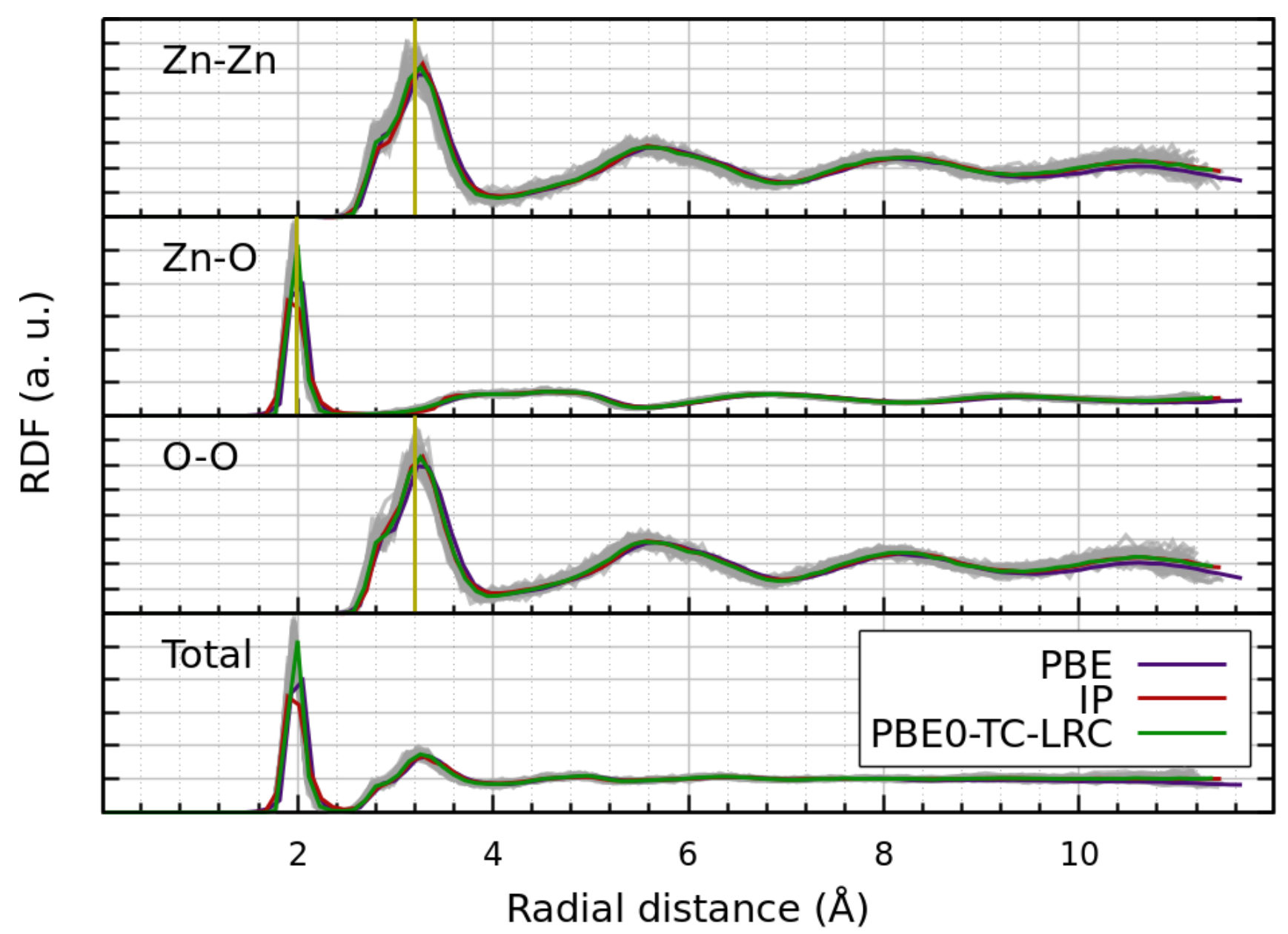

Figure 1: Average radial distribution function from 50 a- $\mathrm{ZnO}$ structures. The steps to generate the amorphous structures is given in Section 2.1. Every structure was fully reoptimized by the PBE0-TC-LRC functional and their average and distribution is shown in green and grey. The calculated IP and PBE profiles ${ }^{[27}$ are also shown as a comparison. Vertical yellow lines represent the wurtzite bulk $\mathrm{ZnO}$ values. 
discussion). The form of this functional is similar to the hybrid HSE (Heyd-Scuzeria-Ernzerhoff) functional. $\frac{62}{62}$ The $37.5 \%$ of the Hartree-Fock (HF) exchange were used to reproduce the experimental c-ZnO band gap within $2 \%$. This amount of exact exchange is commonly used with HSE06 63 to study defects in $\mathrm{ZnO}$ as it reproduces the experimental c-ZnO band gap and the position of its band edges and reliably predicts formation energies and charge transition levels of defects in $\mathrm{ZnO} . \underline{66} \underline{68}$ We use the PBE0-TC-LRC functional in conjunction with the auxiliary density matrix method (ADMM) [69] to reduce the computational cost of the h-DFT calculations.

\subsection{Modeling of polaronic states}

The deficiency of (hybrid) DFT methods in describing electron localization and polaron states is widely recognized. 70 T3 The polaron trapping energies in crystals are usually small $(<0.3 \mathrm{eV})$ and, therefore, even qualitative predictions of polaron states can be inaccurate. Since polaron localization is particularly affected by the self-interaction error inherent in DFT, satisfying the Koopmans' condition $[74$ is often considered as a prerequisite for accurate prediction of polaron stability. 75 The PBE0-TC-LRC functional has two parameters which can be varied to satisfy the Koopmans' condition - the truncation radius and the amount of HF exchange. Here, we used the approach described in refs. 38,76 78. The truncation radius $\mathrm{R}$ was varied to satisfy the Koopmans' condition at two values of Hartree-Fock (HF) exchange of $25 \%$ and $37.5 \%$ so that the following function is minimized: 38

$$
\begin{aligned}
J(R)= & {\left[\varepsilon_{\text {HOMO }}\left(N_{\text {elect }}\right)+E_{\text {gs }}\left(N_{\text {elect }}-1\right)\right.} \\
& \left.-E_{g s}\left(N_{\text {elect }}\right)\right]^{2}+\left[\varepsilon_{\text {HOMO }}\left(N_{\text {elect }}+1\right)\right. \\
& \left.+E_{g s}\left(N_{\text {elect }}\right)-E_{\text {gs }}\left(N_{\text {elect }}+1\right)\right]^{2},
\end{aligned}
$$

where $E_{g s}\left(N_{\text {elect }}\right)$ is the total energy of a ground state (gs) system with $N_{\text {elect }}$ electrons in the perfect neutral system. $\varepsilon_{\text {HOMO }}\left(N_{\text {elect }}\right)$ is the highest occupied molecular orbital (HOMO) of a neutral cell with $N_{\text {elect }}$ electrons. In both cases the minimum of $J(R)$ is at $R=3 \AA$. We note that the linearity condition is satisfied better when a standard value of $25 \%$ of $\mathrm{HF}$ exchange is used. However, at this value the bulk band gap of c- $\mathrm{ZnO}$ is underestimated by $26 \%(2.54 \mathrm{eV})$. 
When using $37.5 \%$ of HF exchange, the band gap is in good agreement with experiment but the Koopmans' condition is satisfied much worse. Both the underestimation of the c-ZnO bulk band gap and the overcorrection of the the linearity condition have been previously reported for the HSE hybrid functional using the same values of HF exchange. ${ }^{75 / 79}$ The dilemma one is facing when trying to satisfy both the band gap and the Koopmans' condition when modeling polaron states has been discussed recently in ref. 80 .

In most of the calculations reported in this paper, the amount of HF exchange was fixed at $37.5 \%$ to match the experimental c-ZnO band gap of $3.44 \mathrm{eV} .8182$ Nevertheless, as we show below, using the standard amount of HF exchange (25\%) does not change our qualitative conclusions.

To test how the h-DFT functional is reproducing the known polaronic states, we calculated the properties of $\mathrm{ZnO}: \mathrm{Li}_{\mathrm{Zn}}$ center, which has been studied experimentally and theoretically. $\frac{83}{88}$ The thermodynamic charge transition level for the $\mathrm{Li}_{\mathrm{Zn}}$ defect, $(\mathrm{Li}-\mathrm{O})^{-/ 0}$, was calculated using the usual expression:

$$
\begin{aligned}
E_{f}= & E_{\text {tot }}^{\text {defect }}-E_{\text {tot }}^{b u l k}+\sum_{i} n_{i} \mu_{i} \\
& +q\left(E_{V B M}+\Delta E_{F}\right),
\end{aligned}
$$

where $E_{\text {tot }}^{\text {dect }}$ and $E_{\text {tot }}^{b u l k}$ are the total energies of the defective and non-defective cells. The change in the number of atoms (positive for additions and negative for removals) and the chemical potential of the removed/added $i$ species are given by $n_{i}$ and $\mu_{i} . E_{V B M}, q$, and $\Delta E_{F}$ are the position of the valence band minimum (VBM), the charge of the defect and a free parameter which accounts for the shifts of the Fermi level, respectively.

The stability of a hole/electron polaron is characterized by its trapping energy, $E_{\text {trap }}$. It is commonly defined (see e.g. refs. 38,89,90) as a difference between the total energies of the system with an extra electron or hole in a perfect geometry state (e.g. perfect crystal) and after the full geometry relaxation. If the fully relaxed state corresponds to a localized charge and is lower in energy than the delocalized state, $E_{\text {trap }}$ corresponds to the thermal ionization energy of the polaron. This definition works well for crystals but is slightly ambiguous for amorphous solids as electronic states of extra electrons or holes in initial unrelaxed state in amorphous structure may be partially localized. Finally, to investigate whether two localized hole states can combine to form 
a bipolaron, we have calculated the interaction of two localized holes in over 20 a- $\mathrm{ZnO}$ structures.

To aid the analysis of electronic states we used an inverse participation ratio (IPR). This parameter is often used to characterize the degree of localization of vibrational and electronic states in amorphous solids. $\frac{38 \mid 91] 96}{96}$. It is particularly convenient when an atom centered basis set is used, as is the case in CP2K. The IPR is calculated as:

$$
\operatorname{IPR}\left(\psi_{n}\right)=\frac{\sum_{i=1}^{N} c_{n_{i}}^{4}}{\left(\sum_{i=1}^{N} c_{n_{i}}^{2}\right)^{2}}
$$

where

$$
\psi_{n}(r)=\sum_{i=1}^{N} c_{n_{i}} \phi_{i}(r)
$$

is a linear combination of atom-centered basis functions, $N$ is the total number of atomic orbitals and $\phi_{i}$ are the basis functions. Using this representation, IPR ranges from 0 to 1 , with a higher IPR values corresponding to localized states, and smaller IPR values corresponding to delocalized states.

Finally, we note that any amorphous structure obtained by freezing a melt may be locally unstable meaning that some local perturbation can cause local structural relaxation. Since electron or hole localization is accompanied by significant distortions in the surrounding amorphous network, they may induce such a perturbation. This is manifested in the fact that, when a charge is removed and the system is allowed to relax, it does not return into its initial state and remains in another (usually lower) energy minimum of neutral amorphous structure. This has been observed, for example, in the case of hole trapping in hydrogenated amorphous silicon. ${ }^{97}$ If this occurs, such a state drops out of distribution of trapping energies caused solely by disorder of local environment. To distinguish such situations we used the following procedure for trapped holes in all 50 a- $\mathrm{ZnO}$ samples: (i) a hole was introduced into the amorphous structure and the system was fully relaxed; (ii) the structure was then made neutral and fully relaxed again; (iii) if the new structure was different from the original one, step (i) was repeated again; (iv) this process is repeated until the neutral structures from two consequent steps are the same. All considered a-ZnO structures were reversible with respect to hole trapping/de-trapping after maximum of two cycles. 


\section{Results and Discussion}

\section{$3.1 \quad$ Properties of $\mathrm{c}-\mathrm{ZnO}$}

The structural characteristics of wurtzite $\mathrm{ZnO}$ as well as the band gap are compared with experimental values in Table 1. The IP calculations reproduce the geometric characteristics better than DFT, but generally the agreement with experiment is quite good. Not unexpectedly, PBE significantly underestimates the band gap.

The left-hand panel of Figure 2 shows the density of states (DoS) of c-ZnO calculated using h-DFT and the super-imposed IPR spectrum. The latter demonstrates that the electronic states are fully delocalized and the bottom of the conduction band has large dispersion. We tried to localize electrons and holes in c-ZnO by e.g. inducing precursor lattice deformation and allowing the charged system to relax, but both extra electron and hole remained delocalized. This is in agreement with previous calculations.

Table 1: Bulk properties of the wurtzite structure of $\mathrm{ZnO}$. Deviations from experiment are shown in parenthesis.

\begin{tabular}{lcccr}
\hline & Experiment 8182 [99] & IP & PBE & PBE0-TC-LRC \\
\hline$a(\AA)$ & 3.2417 & $3.2525(+0.33 \%)$ & $3.2943(+1.62 \%)$ & $3.2120(-0.91 \%)$ \\
$c(\AA)$ & 5.1876 & $5.1979(+0.20 \%)$ & $5.3010(+2.18 \%)$ & $5.1827(-0.09 \%)$ \\
$u$ & 0.3819 & $0.3806(-0.34 \%)$ & $0.3802(-0.45 \%)$ & $0.3791(-0.73 \%)$ \\
$\rho\left(\mathrm{g} / \mathrm{cm}^{3}\right)$ & 5.73 & $5.68(-0.87 \%)$ & $5.42(-5.41 \%)$ & $5.83(+1.75 \%)$ \\
Band gap $(\mathrm{eV})$ & 3.44 & & 0.72 & $3.51(+2.03 \%)$ \\
\hline
\end{tabular}

\section{$3.2 \quad \operatorname{Li}_{\mathrm{Zn}}$ Defect}

To test the h-DFT further, we calculated the experimentally and theoretically well-known $\mathrm{Li}_{\mathrm{Zn}}$ defect in wurtzite $\mathrm{ZnO} .{ }^{75 / 84 \mid 85}$ In this structure, the $\mathrm{Zn}$ cations are four-coordinated with a $\mathrm{Zn}-\mathrm{O}$ distance $d_{\mathrm{Zn}-\mathrm{O}}=1.97 \AA$. Upon substitution of $\mathrm{Zn}$ for $\mathrm{Li}$ ion, there is a strong local atomic relaxation and a localized hole state is formed on one of the neighboring $\mathrm{O}$ ions (see Figure 3). The created $\mathrm{Li}_{\mathrm{Zn}}$ defect shows two different stable configurations: the axial and the nonaxial (Figure 3), where a hole is localized on the axial $\mathrm{O}$ ion or on one of the three equivalent $\mathrm{O}$ ions in the hexagonal 


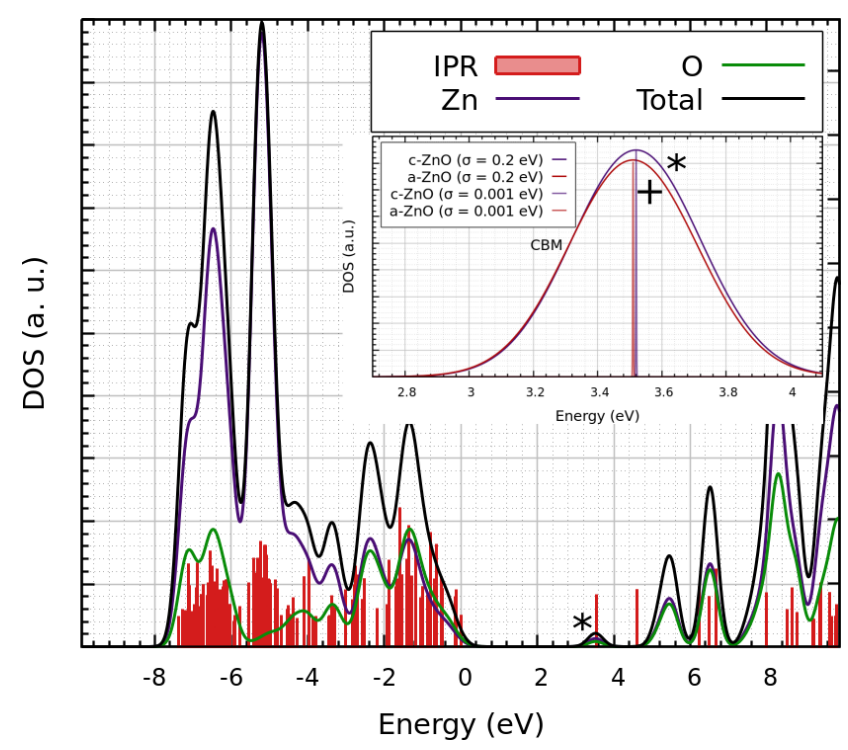

a ) $\mathrm{c}-\mathrm{ZnO}$

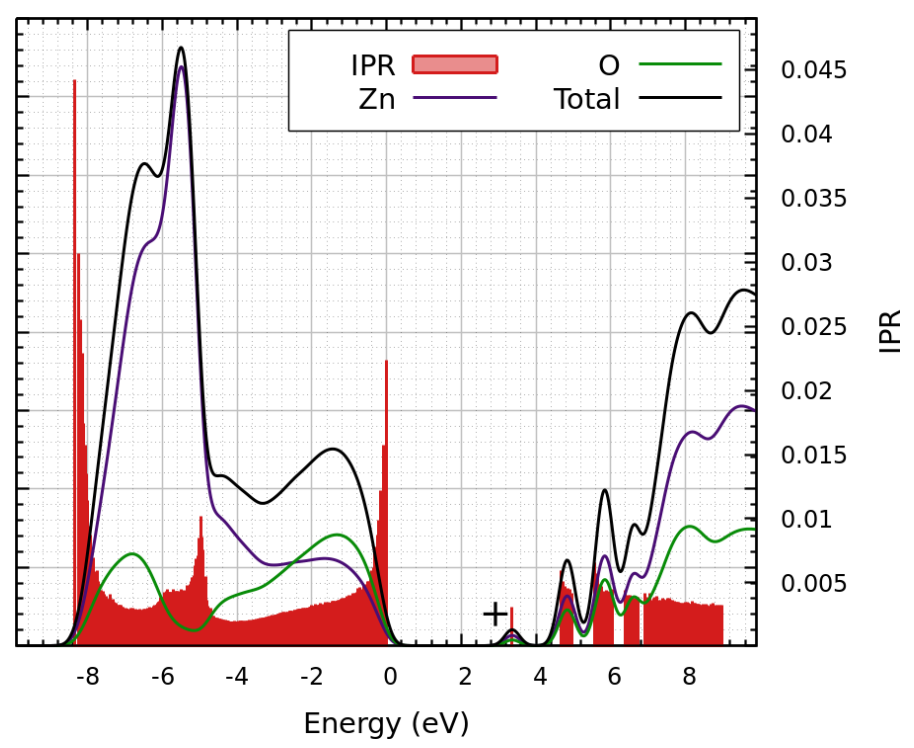

b ) $\mathrm{a}-\mathrm{ZnO}$

Figure 2: DoS for neutrally charged a) wurtzite and b) a-ZnO structures using the PBE0-TC-LRC functional and a Gaussian smearing of $0.20 \mathrm{eV}$. The top of the valence band (VB) was set to $0.0 \mathrm{eV}$. States in the conduction band $(\mathrm{CB})$ were amplified by a factor of 5 to improve visualization. IPR spectra is shown in red. Higher IPR values denote stronger localization. The a-ZnO DoS and IPR plots were averaged from the 50 different amorphous structures. Inset: CBM amplification of $\mathrm{c}-\mathrm{ZnO}\left({ }^{*}\right)$ and a-ZnO $(+)$ with Gaussian smearing of 0.20 and $0.001 \mathrm{eV}$. The profiles in the inset were shifted to help comparison.

sheet, respectively. Both $\mathrm{Li}$ and $\mathrm{O}$ ions undergo strong atomic relaxation, increasing their initial bond distance by $\approx 34 \%$. For the axial $\mathrm{Li}_{\mathrm{Zn}}$ defect, $d_{\|}=2.64 \AA(+34 \%)$ and $d_{\perp}=1.87 \AA$, whereas for the nonaxial defect, $d_{\|}=1.87 \AA$ and $d_{\perp}=2.61 \AA(+34 \%)$. Nonaxial oxygen with no localized hole have the same $d_{\mathrm{Li}-\mathrm{O}}$ as $d_{\|}$of $1.87 \AA$. These structural parameters for the $\mathrm{ZnO}: \mathrm{Li}_{\mathrm{Zn}}$ defect are in excellent agreement with previous theoretical reports by Lany and Zunger ${ }^{\text {[5] }}$ and analysis of the anisotropic Li hfs interaction by Schirmer. ${ }^{84}$. Within the h-DFT accuracy, both configurations are equally stable and are in a good agreement with experiment ${ }^{85}$ where the axial defect was observed to be more stable by only $15(4) \mathrm{meV}$. We note that the PBE functional fails to reproduce this hole polaron, upon Li substitution on Zn site, the hole wavefunction delocalizes across the whole lattice.

Experimentally, the optical absorption of the $\mathrm{ZnO}: \mathrm{Li}_{\mathrm{Zn}}$ defect shows a wide band peak at $\approx 1.5 \mathrm{eV}$, which has been correlated with the $\mathrm{Li}^{+}-\mathrm{O}^{-}$charge state, monitored by electron paramagnetic resonance $(\mathrm{EPR})$. From this peak, using theory for tetrahedral polarons (peak at $8 / 3 E_{p} \approx$ 


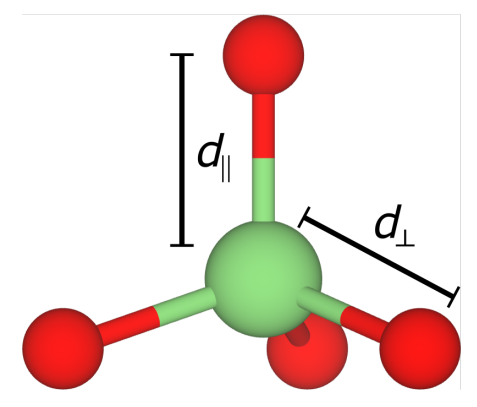

Unrelaxed $0.00 \mathrm{eV}$

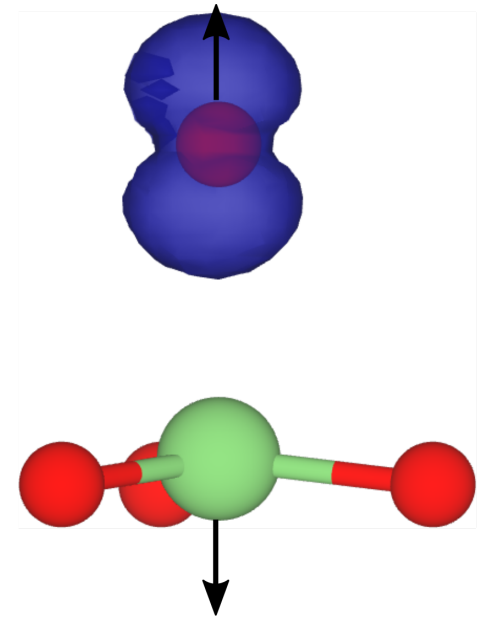

Axial Localised Hole $-0.70 \mathrm{eV}$
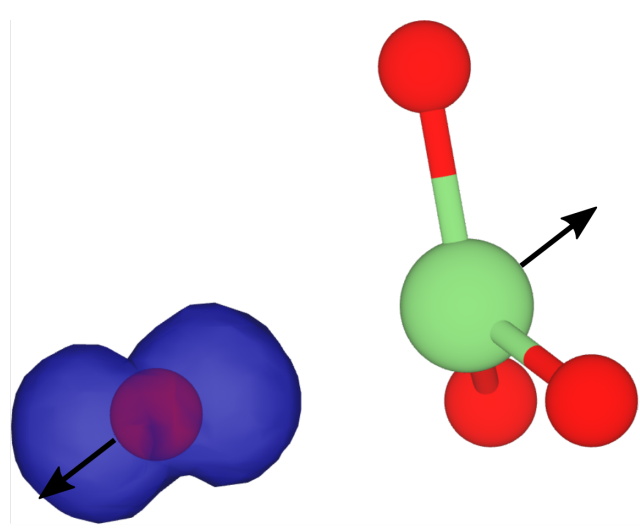

Figure 3: The calculated spin density (blue) and atomic structure of the $\mathrm{Li}_{\text {Zn }}$ defect, before and after atomic relaxation. Isosurface is not shown for the unrelaxed and delocalized defect. After relaxation, the hole is localized either axially or nonaxially, as shown in the figure. The direction of the atomic Li-O relaxation is shown by arrows. Energies with respect to the unrelaxed structure are shown below the figures. Green and red are reserved for Li and O atoms, respectively.

$1.5 \mathrm{eV})$, a polaron stabilization energy $\left(E_{p}\right)$ of $\approx 0.6 \mathrm{eV}$ has been deduced. $\underline{86}$

In this work, trapping energies of 0.70 and $0.71 \mathrm{eV}$ have been calculated for the axial and nonaxial structures. These values are in good agreement with those reported by Schirmer (polaron stabilization energy, $\left.E_{p}\right)$ and Sokol. 100

This defect acts as a deep acceptor with a thermodynamic transition level, $(\mathrm{Li}-\mathrm{O})^{-/ 0}$, of $0.67 \mathrm{eV}$ above of the VBM, which is in agreement with previous studies $\left(0.5 \mathrm{eV}\right.$ to $\left.0.8 \mathrm{eV}^{[101}\right)$.

We conclude that the hybrid PBE0-TC-LRC functional accurately models the electronic structure of non-defective c- $\mathrm{ZnO}$ and the hole polaron formed in the $\mathrm{ZnO}: \mathrm{Li}_{\mathrm{Zn}}$ defect. Therefore, we have chosen this functional to study electron and hole localization in amorphous $\mathrm{ZnO}$.

\subsection{Atomic Structure and Stability of a- $\mathrm{ZnO}$}

The geometries of fifty a-ZnO structures obtained using IPs and the MQ MD procedure described above were further optimized using DFT with the PBE and the PBE0-TC-LRC functionals. The average structural characteristics are summarized in Table 2, In general, there is an excellent agreement between IP and DFT structures, with a topology well defined by IP and preserved after 
the DFT reoptimization. The radial distribution functions are shown in Figure 1. The average a-ZnO $\mathrm{CN}$ is $c a$. 3.9, which is very close to that in c-ZnO. Most of the $\mathrm{Zn}$ and $\mathrm{O}$ species $(85 \%)$ keep a 4-coordinated environment. The remaining ions are 3-coordinated (11\%) and 5-coordinated (4\%). The bond angle distribution (Figure 4 ) is wide among all a- $\mathrm{ZnO}$ structures with a IP (DFT, h-DFT) mean of $109.3^{\circ}\left(109.1^{\circ}, 109.2^{\circ}\right)$ and two peaks at $c a .89^{\circ}$ (rock-salt) and $112^{\circ}$ (wurtzite). There is a density reduction of $11.27,8.3$ and $8.2 \%$ for IP, DFT and h-DFT, respectively, relative to their c-ZnO values - the density distribution with a Gaussian smearing is shown in Figure 5.

The distribution of excess energy, $\Delta E$ (the energy difference per ZnO unit of the amorphous structure with respect to the wurtzite structure) among the 50 different a-ZnO structures is within $0.01 \mathrm{eV}$ for both DFT functionals with an average of 0.32 and $0.38 \mathrm{eV}$ for the PBE and the PBE0TC-LRC functionals, respectively. Similar excess energies have been published for Zn-based oxides $\left(\mathrm{In}_{2} \mathrm{O}_{3}(\mathrm{ZnO}), \mathrm{InGaZnO}_{4}, \mathrm{InAlZnO}_{4}\right) .102$

The energy landscape of every a-ZnO structure was explored using the activation-relaxation technique. In general, the MQ-generated a-ZnO amorphous structures have high structural stability and low propensity for crystallization. We found all structures except one to be stable with respect to local structural re-arrangements generated by the ART algorithm. In one structure, well-defined crystal motifs appeared as a result of structural rearrangements following the ART procedure. In agreement with our previous work, $\stackrel{27}{2}$ these results suggest that stable a-ZnO samples could be produced by fast cooling of a melt.

\subsection{Electronic Structure of a- $\mathrm{ZnO}$}

The electronic structure calculations were carried out for fully h-DFT optimized simulation cells. Figure $2 \mathrm{~b}$ shows the a-ZnO DoS and the IPR spectrum averaged over 50 structures. The top of the VB is mostly composed of $\mathrm{O} 2 p$ orbitals with some $\mathrm{Zn} 3 d$ contributions; whereas $\mathrm{Zn} 4 s$ states dominate at the CBM. When compared to the crystalline phase, amorphous structures show broader peaks, which can be attributed to mixing of the molecular orbitals. The character of the band edges remains the same as in $\mathrm{c}-\mathrm{ZnO}$.

We note that several peaks above $2 \mathrm{eV}$ in Figure 2 for both c-ZnO and a-ZnO are part of the 


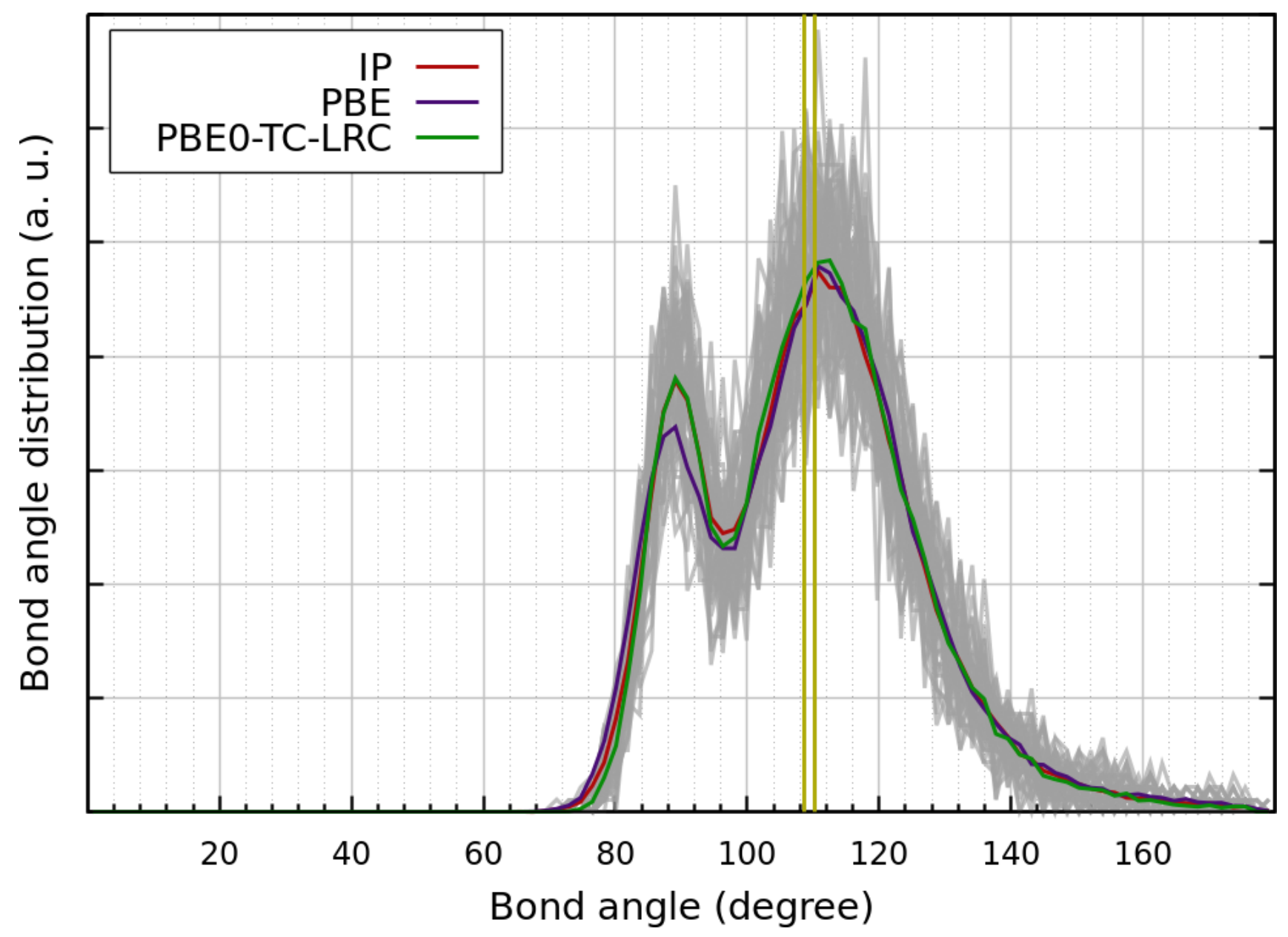

Figure 4: The $\mathrm{Zn}-\mathrm{O}-\mathrm{Zn}$ bond angle distribution, from $50 \mathrm{a}-\mathrm{ZnO}$ structures, calculated with the PBE0-TC-LRC functional is shown in grey. Average profiles for fully optimized IP, PBE ${ }^{27}$ and PBE0-TC-LRC structures are also displayed. Vertical green lines represent the wurtzite bulk $\mathrm{ZnO}$ values: $\approx 108^{\circ}$ for two $\mathrm{Zn}$ in different hexagonal planes and $\approx 110^{\circ}$ for two $\mathrm{Zn}$ in the same hexagonal sheet. 


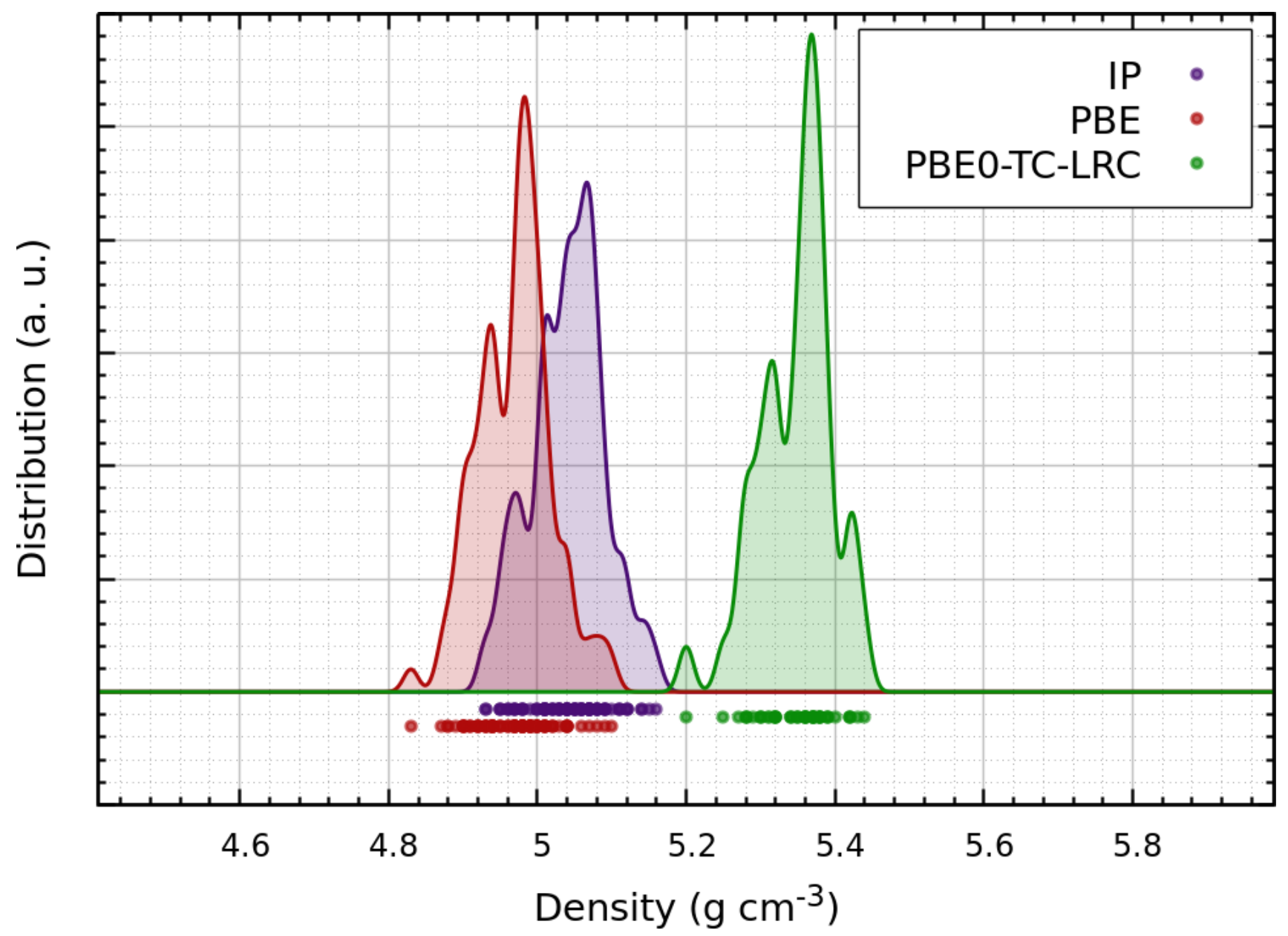

Figure 5: Density distribution (with a Gaussian smearing of $\sigma=0.01 \mathrm{~g} \mathrm{~cm}^{-3}$ ), from 50 structures, of the PBE0-TC-LRC a-ZnO structures. IP and DFT distributions ${ }^{27}$ are also shown for comparison. The wurtzite bulk value at $298 \mathrm{~K}$ is $5.6 \mathrm{~g} \mathrm{~cm}^{-3}$.103 
Table 2: The average numbers of 3,4 and 5-coordinated atoms (in percentage) and density ( $\rho$, in $\mathrm{g} / \mathrm{cm}^{3}$ ) for 324-atom a-ZnO structures calculated using IPs and two density functionals. The number of samples is 50 for each column. The average excess energy, $\Delta E$ in eV, and its standard deviation is also shown. The average $\mathrm{CN}$ for $\mathrm{Zn}$ and $\mathrm{O}$ species is given by $n_{Z n}$ and $n_{O}$. Calculated averages are after a full optimization.

\begin{tabular}{lcccc}
\hline Type & CN & IP & PBE & PBE0-TC-LRC \\
\hline $\mathrm{Zn}$ & 3 & 11.9 & 12.1 & 12.1 \\
& 4 & 85.0 & 84.6 & 85.8 \\
& 5 & 3.0 & 3.2 & 2.1 \\
$\mathrm{O}$ & 3 & 12.6 & 12.5 & 11.6 \\
& 4 & 83.7 & 83.9 & 86.8 \\
& 5 & 3.7 & 3.6 & 1.6 \\
$\Delta E$ & & 0.50 & 0.32 & 0.38 \\
$\Delta E$ std dev & & 0.02 & 3.91 & 0.01 \\
$n_{Z n}$ & & 3.91 & 3.91 & 3.90 \\
$n_{O}$ & 3.91 & 4.97 & 3.90 \\
$\rho$ & & 5.05 & & 5.35 \\
\hline
\end{tabular}

CB. The apparent discontinuity of the DoS results from the fact that our $\Gamma$-point calculations in 300 atom cell of c-ZnO do not account for enough $k$ points. The c-ZnO DoS calculations [53] using a dense $k$-mesh of $9 \times 9 \times 7$ and a hybrid PBEsol0 functional show a continuous DoS. A test, using cell sizes 8 times bigger (2400 and 2592 atoms for c-ZnO and a-ZnO) than the ones shown in Figure 2 and the GGA PBE functional, showed no splitting at the CBM and a similar behaviour of $\mathrm{c}-\mathrm{ZnO}$ and $\mathrm{a}-\mathrm{ZnO}$ around the bottom of the $\mathrm{CB}$. The DoS characteristics across all 50 a- $\mathrm{ZnO}$ samples are similar with no states splitting into the gap due to disorder. Therefore, no substantial detrimental effect on the optical transparency of a- $\mathrm{ZnO}$ is expected.

In a semiconductor, the effective mass, which is directly related to band dispersion, can be calculated from the shape of the DoS near to VB and CB edges (see, e.g., Section 4.6 and Eq. 4.33 in ref 104). Therefore, the small differences between the c- $\mathrm{ZnO}$ and a- $\mathrm{ZnO}$ close to the CBM suggest that dispersion is not severely affected upon amorphization (see inset in Figure 2a). In contrast, the a-ZnO $\mathrm{VB}$ is much less disperse than in c-ZnO. We note that similar effects upon amorphization on the effective mass have been reported for other Zn-based semiconductors. $102[105 \mid 106]$ The high dispersion on the a-ZnO CB is consistent with the IPR spectra in Figure $2 \mathrm{~b}$ and the delocalized nature of the lowest unoccupied molecular orbital (LUMO) illustrated in Figure 6. It 
is characteristic to other materials with a s-band CB character (see refs. 105107 and references therein).

For a-ZnO, the IPR spectrum exhibits well localized states at the VBM and no localization at the CBM (Figure 2b). Upon amorphization, the intrinsic $n$-type conductivity in $\mathrm{ZnO}$ will not be substantially affected by the presence of "heavy" electrons at the CB. On the other hand, some degree of localization of VBM states suggests that disorder can lead to hole localization upon structural relaxation. Similar asymmetry in the character of localization of the a-ZnO VBM and CBM states has also been seen in other amorphous oxides, such as $\operatorname{In}_{2} \mathrm{O}_{3}, \mathrm{IGZO}, \mathrm{Al}_{2} \mathrm{O}_{3} \cdot \frac{21|38| 105 / 106 / 108}{110}$

The average a- $\mathrm{ZnO}$ band gap is $c a .3 .36 \mathrm{eV}$, e.g. $0.15 \mathrm{eV}$ lower than that in the crystalline phase, with a standard deviation of $0.08 \mathrm{eV}$. The latter is in line with experiments, 111 where oxides

with large cations $\left(r_{i} \gtrsim 0.7 \AA\right)$ show no significant band gap difference between the crystalline and amorphous phase, whereas gap widening is observed for more compact cations. For instance, in $\mathrm{Al}_{2} \mathrm{O}_{3}$, hybrid DFT band gap goes from 5.5 to $8.6 \mathrm{eV}$ as a result of oxide crystallization. 381111

\subsection{Electron and hole trapping in a- $\mathrm{ZnO}$}

To the best of our knowledge, no electron or hole polaron formation has been observed in c- $\mathrm{ZnO}$. This is corroborated by the IPR spectrum of c-ZnO in Figure 2a, which shows that all states in the valence and conduction bands are delocalized. However, the IPR spectrum of a-ZnO in Figure 2b exhibits several states with different degree of localization near VBM whereas the conduction band states remain delocalized. The character of one-electron wavefunctions of these a- $\mathrm{ZnO}$ states is illustrated in Figure 6. Extra electrons indeed remain delocalized in a-ZnO. In contrast, extra holes added into a-ZnO cells localize spontaneously in every structure upon the geometry optimization.

\subsubsection{Structure of localized holes}

Holes in a-ZnO localize in two different configurations: i) on $\mathrm{O} 2 p$ orbitals of one or two $\mathrm{O}$ ions; and ii) on Zn $3 d$ and O $2 p$ orbitals; both configurations are shown in Figure 7 . From fifty 324-atom a-ZnO structures, $70 \%(16 \%, 2 \%)$ of the holes sit in $p$ orbitals of 3 -coordinated (4-coordinated, 2-coordinated) oxygen ions, the remaining $12 \%$ holes are shared by 5 ions: one $\mathrm{Zn}$ ( $3 d$ orbitals) and 


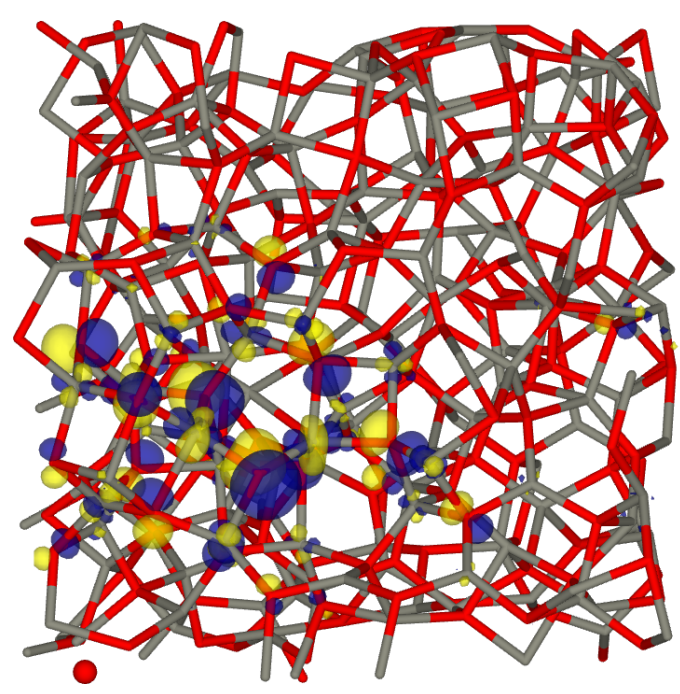

a ) $\mathrm{HOMO}$

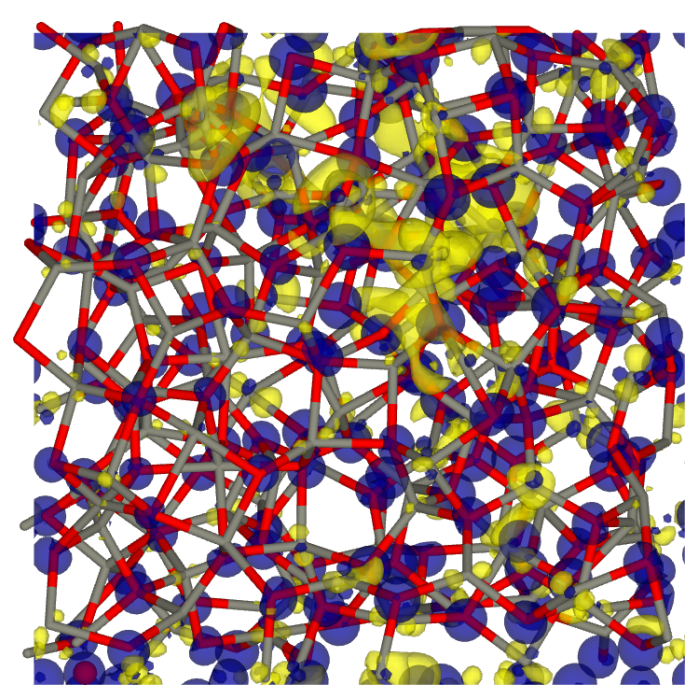

b ) LUMO

Figure 6: The wavefunctions of the HOMO and LUMO of a-ZnO. Grey is reserved for $\mathrm{Zn}$ atoms. Blue and yellow indicate positive and negative isosurfaces.

its 4 adjacent $\mathrm{O}$ atoms. In the case of hole localization on $\mathrm{O}$ ions, in $92 \%$ of cases it is localized on one $\mathrm{O}$ ion and in $8 \%$ delocalized by two $\mathrm{O}$ ions. As discussed earlier, the $\mathrm{VBM}$ of c-ZnO and a-ZnO is mainly comprised from $\mathrm{O} 2 p$ states with some contribution from $\mathrm{Zn} 3 d$ states. This explains why the hole localization involves $\mathrm{Zn}$ atoms in $12 \%$ of the structures. The geometry of these hole states is planar-like in every structure, as seen in Figure 7. An example of the structural changes upon full geometry relaxation of the most common a-ZnO hole polaron is shown in Figure 9.

\subsubsection{Trapping energies of localized holes}

Hole trapping energies range from 0.59 to $1.30 \mathrm{eV}$, with an average of $0.87 \mathrm{eV}$ and a standard deviation of $0.17 \mathrm{eV}$. The distribution of trapping energies shown in Figure 8 spans over $0.7 \mathrm{eV}$. On average, the deeper traps correspond to 2-coordinated $\mathrm{O}$ ions $(1.3 \mathrm{eV})<3$-coordinated Oions $(0.9 \mathrm{eV})<4$-coordinated O-ions $(0.71 \mathrm{eV})<$ configurations involving Zn ions $(0.7 \mathrm{eV})$. The a-ZnO IPR spectrum in Figure 2b shows four states at VBM with higher degree of localization. Correspondingly, on average, there are 3-4 precursor sites in each 324 -atom cell $\left(9.78 \times 10^{20} \mathrm{~cm}^{-3}\right)$.

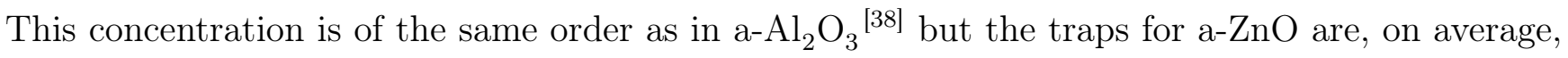
$70 \%$ shallower.

We note that the calculated trapping energies are affected by several factors with respect to 
similar calculations in the crystal phase. Since they are calculated as total energy differences between the initial hole state in the a-ZnO structure and the fully relaxed state, they are affected by the fact that the initial hole states are already partially localized, as illustrated in Figures $6 a$ and 9a, but the degree of localization differs in different a- $\mathrm{ZnO}$ samples. The greater difference in the degree of localization between an unrelaxed and relaxed charged state results in a higher kinetic energetic cost upon localization. Measuring the trapping energies with respect to the hole mobility edge, which is located about $0.5 \mathrm{eV}$ deeper with respect to the VBM (see Figure 2b), would reduce the trapping energies.

The second significant factor is the amount of HF exchange. As discussed earlier, the $37.5 \%$ of the $\mathrm{HF}$ exchange selected to reproduce the experimental c-ZnO band gap overcorrects the functional's linearity, whereas the standard $25 \%$ of HF exchange satisfies the Koopmans' condition but underestimates the c-ZnO band gap. Therefore, we have recalculated the hole trapping energies for every a-ZnO structure using the standard $25 \% \mathrm{HF}$ exchange, with the distribution of trapping energies shown in Figure 8. Qualitatively, the results remain the same but the trapping energies are on average lower when less HF exchange is used. Hole trapping energies using 37.5\% (25\%) exchange are, on average, $0.87 \mathrm{eV}(0.52 \mathrm{eV})$. The standard deviation of trapping energies are, nevertheless, similar reflecting the distribution of local environments of structural precursor sites. The fact that holes remain localized in a-ZnO at two significantly different values of the $\mathrm{HF}$ exchange provides additional confidence that a-ZnO structures can trap holes.

Yet another factor affecting the distribution of hole trapping energies concerns the fact that simulation cells have different PBE0-TC-LRC densities ranging from 5.20 to $5.44 \mathrm{~g} / \mathrm{cm}^{3}$. The physical meaning of distribution of densities in a-ZnO structures has been discussed earlier. 27 The ensemble of relatively small 324-atom simulation cells reflects the local density variations in different regions of a macroscopic sample, as shown in ref. 27 using a 768000-atom cell. From this point of view, the distribution of trapping energies accounts for traps located in different regions of an a-ZnO sample. There is no correlation between the charge trapping energies and the density of the simulation cell.

Finally we note that the so-called "reversibility" also affects the values and distribution of 


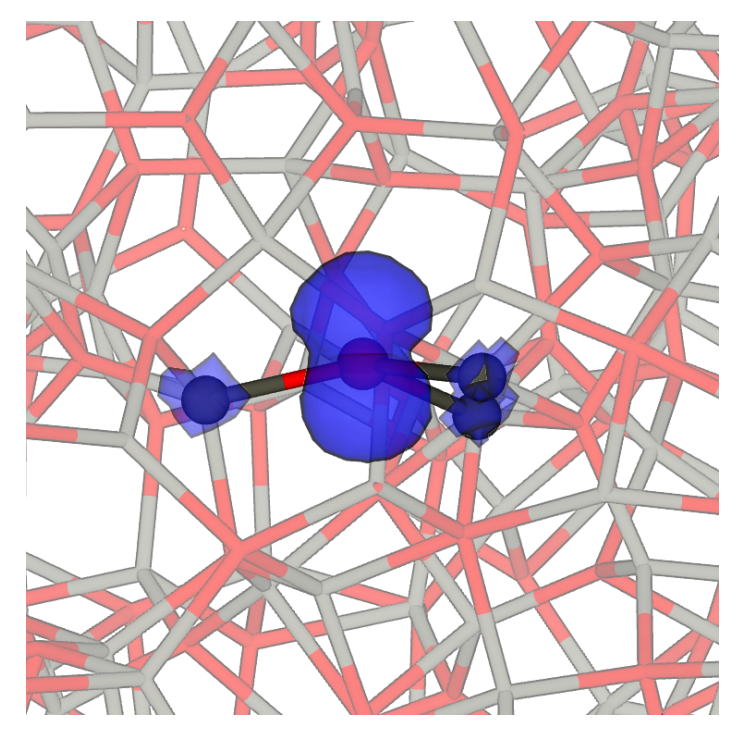

a ) Hole localized on an $\mathrm{O} p$ orbital

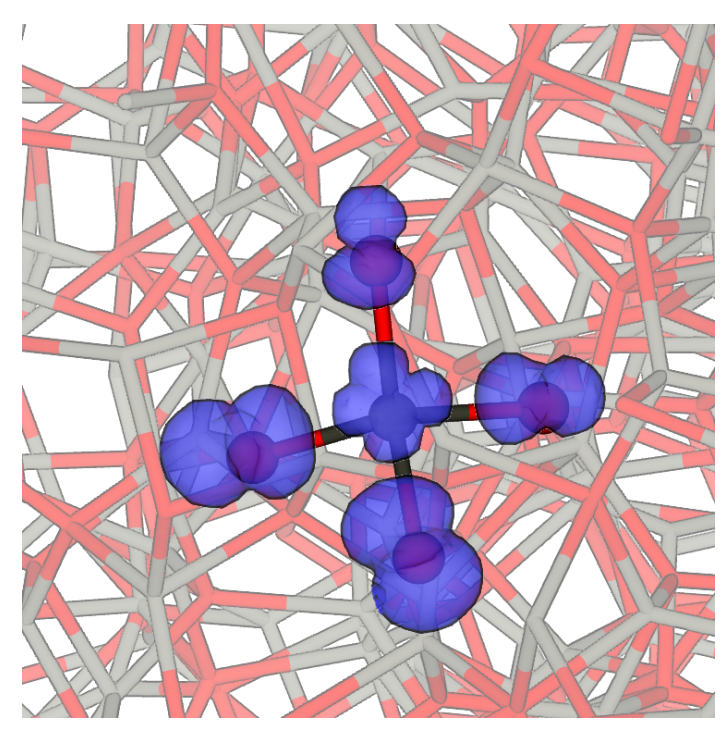

b ) Hole localized on $\mathrm{Zn} d$ and $\mathrm{O} p$ orbitals

Figure 7: The spin density of the hole polarons (blue) in a-ZnO. a) The hole is centered in an undercoordinate $\mathrm{O}$ ion. $\mathrm{b}$ ) The hole is centered in a 4-coordinated $\mathrm{Zn} d$ orbital and its adjacent $\mathrm{O}$ ions.

trapping energies shown in Figure 8. In a crystal, hole trapping/detrapping can be repeated without inducing any structural changes. Our calculations show that hole configurations with high trapping energies in a- $\mathrm{ZnO}$ lead to significant changes in the amorphous structure. In some cases, when the missing electron is injected into such structures, the a-ZnO structure does not return into its previous state but remains in a different minimum of the energy landscape. Adding a hole back typically leads to smaller trapping energies. This means that the original high trapping energy includes the gain in energy due to the structural rearrangement. From the $50 \mathrm{a}-\mathrm{ZnO}$ structures, 16 samples were found to be irreversible after the first cycle (shown in Figure 8 with arrows), with only 1 showing strong atomic distortions between the two local minima. After the first cycle, most of the irreversible cases (12) find a shallower hole trap, whereas the rest have deeper traps in the second cycle. In our a-ZnO simulation cells, there are more than one precursor sites that can localize a hole, in 9 out of the 16 irreversible cases, the hole polaron in the second cycle sits in a different site. All structures were found to be reversible after the second cycle. Similar results have been obtained for hole traps in hydrogenated amorphous silicon. 97 


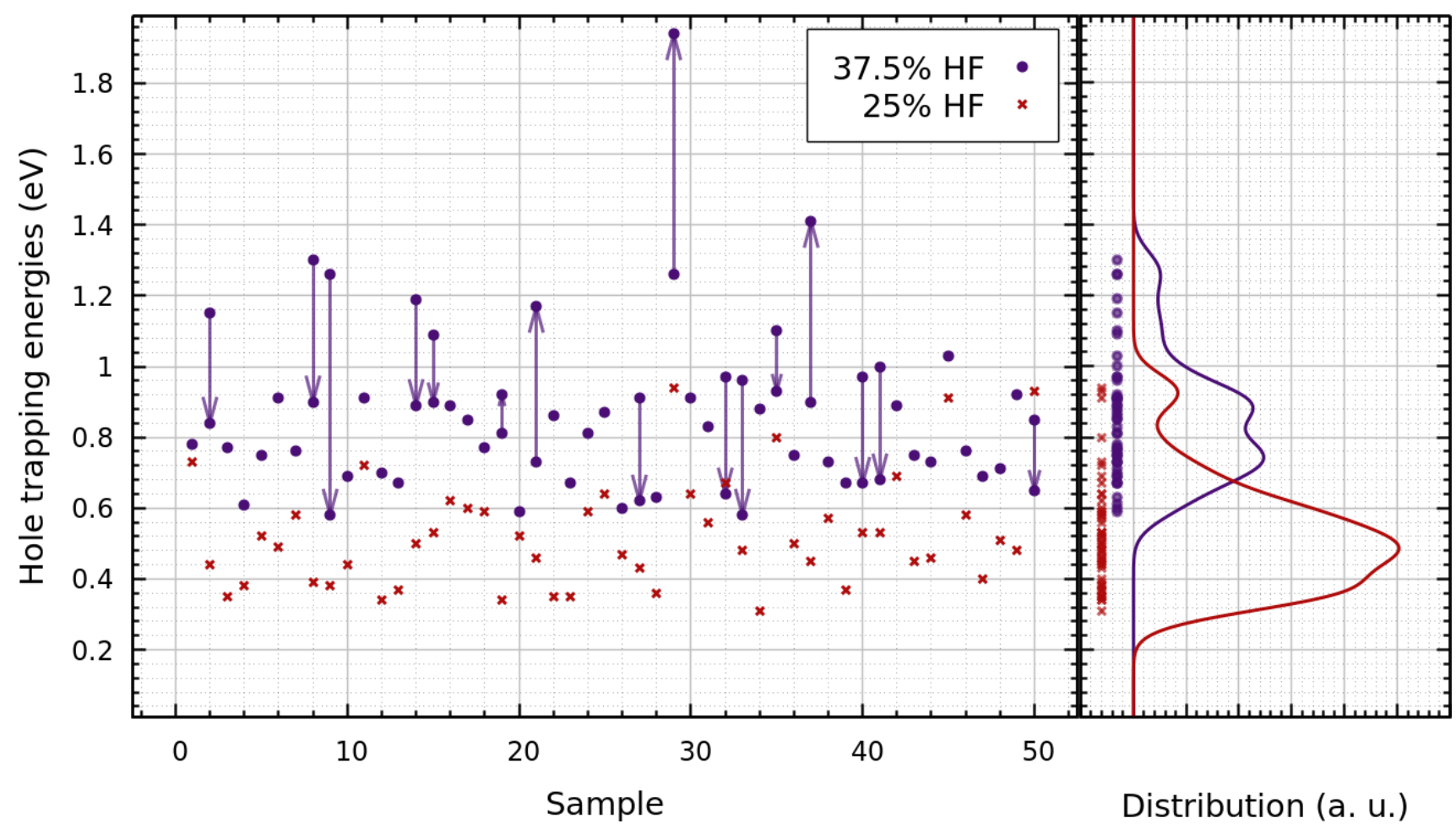

Figure 8: Calculated hole trapping energies for 50 structures using $37.5 \%$ and $25 \%$ of the exact HF exchange and 324-atom simulation boxes. The 16 irreversible cases are shown with arrows. The Gaussian smeared charge trapping energy distribution is shown on the right with a $\sigma=0.05 \mathrm{eV}$.

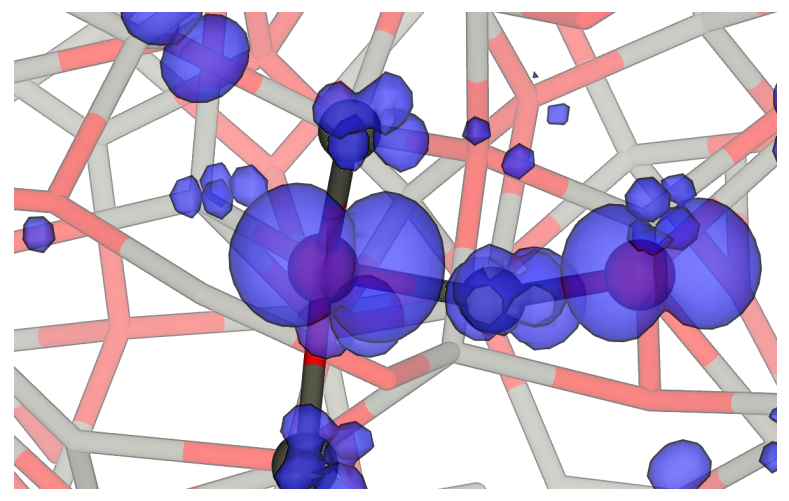

a ) Spin density before optimization

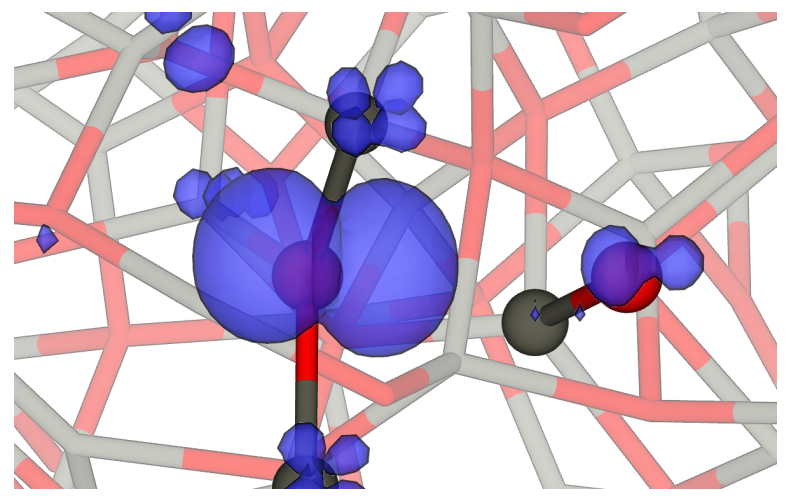

b ) Spin density after optimization

Figure 9: An example of the the spin density of the most common hole polaron in a- $\mathrm{ZnO}$. a) Before structural optimization, and b) after the full geometry relaxation.

\subsubsection{Interaction between localized holes}

Binding of localized holes and formation of bipolarons has been suggested in several metal oxides, such as $\mathrm{HfO}_{2}, \mathrm{Al}_{2} \mathrm{O}_{3}$, anatase $\mathrm{TiO}_{2}, \mathrm{~V}_{2} \mathrm{O}_{5}, \mathrm{MoO}_{3}$, InGaZnO, etc. ${ }^{110112114]}$ In all cases hole bipolarons are stabilized by forming a bond between the two adjacent $\mathrm{O}$ ions each carrying a hole. The energy gain due to this bond formation has to exceed both the hole-hole Coulomb repulsion 
and the cost of large distortion of the surrounding amorphous network. We note that, the oxides mentioned above all share relatively short $\mathrm{O}-\mathrm{O}$ distances, which facilitates the $\mathrm{O}-\mathrm{O}$ bond formation upon hole trapping. However, in a- $\mathrm{ZnO}$ the $\mathrm{O}-\mathrm{O}$ separation is, on average, about $30 \%$ larger than in those oxides, which implies a much higher energetic penalty for $\mathrm{O}-\mathrm{O}$ binding and the accompanying structural distortions. This is the most likely reason why no pairing of localized holes was observed in a-ZnO. Two localized holes prefer to remain separated in all a-ZnO structures considered.

\section{Summary and Conclusions}

To summarize, the results of h-DFT calculations predict hole localization in a-ZnO structures, but no electron localization. A total of 50 a- $\mathrm{ZnO}$ structures, consisting of 324 atoms, were created using a MD MQ technique with the potential energy landscape described by accurate IPs. Both atomic positions and lattice parameters were fully optimized using the PBE and PBE0-TC-LRC density functionals. The hybrid PBE0-TC-LRC functional was tested for compliance with the generalized Koopmans' condition and was used with both $37.5 \%$ and $25 \%$ HF exchange. This functional reproduces the localized hole states in the well-known $\mathrm{Li}_{\mathrm{Zn}}$ defect in $\mathrm{c}-\mathrm{ZnO}$ and its thermodynamic (0/-) transition level. The character of the band edges of a-ZnO remains the same as in c-ZnO: $\mathrm{VB}$ consists of $\mathrm{O} 2 p$ orbitals and some $\mathrm{Zn} 3 d$ contributions, whereas $\mathrm{Zn} 4 s$ states are predominant at the CBM. The a-ZnO VB is less disperse than that in $\mathrm{c}-\mathrm{ZnO}$ and IPR spectrum exhibits localized states at the band edge. The dispersion in the a-ZnO CB remains similar to that in c-ZnO and no localized states were predicted by the IPR analysis. Calculations of extra electron and holes in c-ZnO demonstrate no localization. However, h-DFT calculations predict stable hole trapping in a-ZnO for both values of $\mathrm{HF}$ exchange employed. Holes localize predominantly on lowcoordinated oxygen ions with trapping energies ranging from 0.57 to $1.30 \mathrm{eV}$ averaging at $0.87 \mathrm{eV}$. The trapping energy is larger for lowest coordinated $\mathrm{O}$ sites in a-ZnO. No pairing of two localized holes was found in a- $\mathrm{ZnO}$.

The average a- $\mathrm{ZnO}$ band gap is larger than $3 \mathrm{eV}$ with no defect states in the middle of the band gap, which accounts for a good transparency. Electron conductivity of a-ZnO can be controlled 
by doping, as in c-ZnO. High dispersion of the $\mathrm{CB}$ maintained upon amorphization (see inset in Figure 2a should provide high mobility of electrons in the CB. Therefore, a- $\mathrm{ZnO}$ is expected to remain a good $n$-type TCO. The presence of hole traps in a- $\mathrm{ZnO}$ could, however, improve the performance of this material in other applications, such as photo-catalysis, where exciton recombination is highly undesirable.

\section{Acknowledgements}

We acknowledge funding provided by EPSRC under grants EP/K01739X/1 and EP/P013503/1, and by Leverhulme Trust grant RPG-2016-135. Computer facilities on the ARCHER UK National Supercomputing Service have been provided via the UKs HPC Materials Chemistry Consortium (EPSRC Grant No. EP/L000202). This work used the UK Materials and Molecular Modelling Hub for computational resources, which is partially funded by EPSRC (EP/P020194). The authors acknowledge the use of the UCL Grace High Performance Computing Facility (Grace@UCL), and the associated support services, in the completion of this work. The authors thank J. Cottom, D. Z. Gao, J. Strand, A. A. Sokol, N. Mousseau and H. Hosono for helpful discussions.

\section{References}

[1] C. G. Granqvist, Sol. Energy Mater. Sol. Cells 2007, 91, 1529.

[2] A. Klein, C. Körber, A. Wachau, F. Säuberlich, Y. Gassenbauer, S. P. Harvey, D. E. Proffit, T. O. Mason, Materials (Basel). 2010, 3, 4892.

[3] D. S. Ginley, C. Bright, MRS Bull. 2000, 25, 15.

[4] B. G. Lewis, D. C. Paine, MRS Bull. 2000, 25, 22.

[5] H. E. A. Huitema, G. H. Gelinck, J. B. P. H. van der Putten, K. E. Kuijk, C. M. Hart, E. Cantatore, P. T. Herwig, A. J. J. M. van Breemen, D. M. de Leeuw, Nature 2001, 414, 599. 
[6] J. S. Park, W.-J. Maeng, H.-S. Kim, J.-S. Park, Thin Solid Films 2012, 520, 1679.

[7] E.-G. Fu, D.-M. Zhuang, G. Zhang, Z. Ming, W.-F. Yang, J.-J. Liu, Microelectronics J. 2004, 35, 383.

[8] W. L. Bragg, J. A. Darbyshire, Trans. Faraday Soc. 1932, 28, 522.

[9] A. B. M. A. Ashrafi, A. Ueta, A. Avramescu, H. Kumano, I. Suemune, Y.-W. Ok, T.-Y. Seong, Appl. Phys. Lett. 2000, 76, 550.

[10] S.-K. Kim, S.-Y. Jeong, C.-R. Cho, Appl. Phys. Lett. 2003, 82, 562.

[11] Ü. Özgür, Y. I. Alivov, C. Liu, A. Teke, M. A. Reshchikov, S. DoÄ§an, V. Avrutin, S.-J. Cho, H. Morkoç, J. Appl. Phys. 2005, 98, 041301.

[12] C. R. A. Catlow, S. A. French, A. A. Sokol, A. A. Al-Sunaidi, S. M. Woodley, J. Comput. Chem. 2008, 29, 2234.

[13] Z. Xingwen, L. Yongqiang, L. Ye, L. Yingwei, X. Yiben, Vacuum 2006, 81, 502.

[14] J. M. Khoshman, M. E. Kordesch, Thin Solid Films 2007, 515, 7393.

[15] H.-H. Hsieh, C.-C. Wu, Appl. Phys. Lett. 2007, 91, 013502.

[16] S. Hayamizu, H. Tabata, H. Tanaka, T. Kawai, J. Appl. Phys. 1996, 80, 787.

[17] K. S. Hwang, Y. J. Lee, S. Hwangbo, J. Ceram. Process. Res. 2007, 8, 305.

[18] B. S. Zou, V. V. Volkov, Z. L. Wang, Chem. Mater. 1999, 11, 3037.

[19] S. T. Tan, B. J. Chen, X. W. Sun, W. J. Fan, H. S. Kwok, X. H. Zhang, S. J. Chua, J. Appl. Phys. 2005, 98, 013505.

[20] R. Raghavan, M. Bechelany, M. Parlinska, D. Frey, W. M. Mook, A. Beyer, J. Michler, I. Utke, Appl. Phys. Lett. 2012, 100, 191912.

[21] A. Pandey, H. Scherich, D. Drabold, J. Non. Cryst. Solids 2017, 455, 98. 
[22] D. Tahaö̈ßlu, M. Durandurdu, J. Non. Cryst. Solids 2018, 481, 27.

[23] A. Roy, Y.-T. Cheng, M. L. Falk, J. Phys. Chem. C 2016, 120, 2529.

[24] K.-H. Lin, S.-J. Sun, S.-P. Ju, J.-Y. Tsai, H.-T. Chen, J.-Y. Hsieh, J. Appl. Phys. 2013, 113, 073512.

[25] D. Muñoz Ramo, A. Chroneos, M. Rushton, P. Bristowe, Thin Solid Films 2014, 555, 117.

[26] H.-X. Deng, S.-H. Wei, S.-S. Li, J. Li, A. Walsh, Phys. Rev. B 2013, 87, 125203.

[27] D. Mora-Fonz, A. L. Shluger, Phys. Rev. B 2019, 99, 014202.

[28] N. Mousseau, G. T. Barkema, Phys. Rev. B 2000, 61, 1898.

[29] E. Machado-Charry, L. K. Béland, D. Caliste, L. Genovese, T. Deutsch, N. Mousseau, P. Pochet, J. Chem. Phys. 2011, 135, 034102.

[30] N. Mousseau, G. T. Barkema, Phys. Rev. E 1998, 57, 2419.

[31] G. T. Barkema, N. Mousseau, Phys. Rev. Lett. 1996, 77, 4358.

[32] H. Kallel, N. Mousseau, F. Schiettekatte, Phys. Rev. Lett. 2010, 105, 045503.

[33] L. Zhong, J. Wang, H. Sheng, Z. Zhang, S. X. Mao, Nature 2014, 512, 177.

[34] Y. Q. Wang, W. D. Chen, X. B. Liao, Z. X. Cao, Nanotechnology 2003, 14, 1235.

[35] E. Leon-Gutierrez, A. Sepúlveda, G. Garcia, M. T. Clavaguera-Mora, J. Rodríguez-Viejo, Phys. Chem. Chem. Phys. 2010, 12, 14693.

[36] P. Luo, C. R. Cao, F. Zhu, Y. M. Lv, Y. H. Liu, P. Wen, H. Y. Bai, G. Vaughan, M. di Michiel, B. Ruta, W. H. Wang, Nat. Commun. 2018, 9, 1389.

[37] A.-M. El-Sayed, M. B. Watkins, V. V. Afanas'ev, A. L. Shluger, Phys. Rev. B 2014, 89, 125201.

[38] O. A. Dicks, A. L. Shluger, J. Phys. Condens. Matter 2017, 29, 314005. 
[39] M. Kaviani, J. Strand, V. V. Afanas'ev, A. L. Shluger, Phys. Rev. B 2016, 94, 020103.

[40] A. L. Linsebigler, G. Lu, J. T. Yates, Chem. Rev. 1995, 95, 735.

[41] C. Soci, A. Zhang, B. Xiang, S. A. Dayeh, D. P. R. Aplin, J. Park, X. Y. Bao, Y. H. Lo, D. Wang, Nano Lett. 2007, 7, 1003.

[42] H. Sezen, H. Shang, F. Bebensee, C. Yang, M. Buchholz, A. Nefedov, S. Heissler, C. Carbogno, M. Scheffler, P. Rinke, C. Wöll, Nat. Commun. 2015, 6, 6901.

[43] H. Zhang, R. Zong, Y. Zhu, J. Phys. Chem. C 2009, 113, 4605.

[44] N. Jones, B. Ray, K. T. Ranjit, A. C. Manna, FEMS Microbiol. Lett. 2008, $279,71$.

[45] K. R. Raghupathi, R. T. Koodali, A. C. Manna, Langmuir 2011, 27, 4020.

[46] A. Sirelkhatim, S. Mahmud, A. Seeni, N. H. M. Kaus, L. C. Ann, S. K. M. Bakhori, H. Hasan, D. Mohamad, Nano-Micro Lett. 2015, 7, 219.

[47] S. Ostrovsky, G. Kazimirsky, A. Gedanken, C. Brodie, Nano Res. 2009, 2, 882.

[48] P. Mitra, D. Dutta, S. Das, T. Basu, A. Pramanik, A. Patra, ACS Omega 2018, 3, 7962.

[49] P. Sivakumar, M. Lee, Y.-S. Kim, M. S. Shim, J. Mater. Chem. B 2018, 6, 4852.

[50] J. F. Cordaro, Y. Shim, J. E. May, J. Appl. Phys. 1986, 60, 4186.

[51] L. Whitmore, A. A. Sokol, C. A. Catlow, Surf. Sci. 2002, 498, 135.

[52] S. Plimpton, J. Comput. Phys. 1995, 117, 1.

[53] D. Mora-Fonz, J. Buckeridge, A. J. Logsdail, D. O. Scanlon, A. A. Sokol, S. Woodley, C. R. A. Catlow, J. Phys. Chem. C 2015, 119, 11598.

[54] D. Mora-Fonz, T. Lazauskas, M. R. Farrow, C. R. A. Catlow, S. M. Woodley, A. A. Sokol, Chem. Mater. 2017, 29, 5306. 
[55] D. Mora-Fonz, T. Lazauskas, S. M. Woodley, S. T. Bromley, C. R. A. Catlow, A. A. Sokol, J. Phys. Chem. C 2017, 121, 16831.

[56] S. Le Roux, P. Jund, Comput. Mater. Sci. 2010, 49, 70.

[57] J. VandeVondele, M. Krack, F. Mohamed, M. Parrinello, T. Chassaing, J. Hutter, Comput. Phys. Commun. 2005, 167, 103.

[58] J. P. Perdew, K. Burke, M. Ernzerhof, Phys. Rev. Lett. 1996, 77, 3865.

[59] J. VandeVondele, J. Hutter, J. Chem. Phys. 2007, 127, 114105.

[60] S. Goedecker, M. Teter, J. Hutter, Phys. Rev. B 1996, 54, 1703.

[61] M. Guidon, J. Hutter, J. VandeVondele, J. Chem. Theory Comput. 2009, 5, 3010.

[62] J. Heyd, G. E. Scuseria, M. Ernzerhof, J. Chem. Phys. 2006, 124, 219906.

[63] Y. K. Frodason, K. M. Johansen, T. S. Bjørheim, B. G. Svensson, A. Alkauskas, Phys. Rev. $B$ 2017, 95, 094105.

[64] F. Oba, M. Choi, A. Togo, I. Tanaka, Sci. Technol. Adv. Mater. 2011, 12, 034302.

[65] A. Alkauskas, Q. Yan, C. G. Van de Walle, Phys. Rev. B 2014, 90, 075202.

[66] F. Oba, A. Togo, I. Tanaka, J. Paier, G. Kresse, Phys. Rev. B 2008, 77, 245202.

[67] J. L. Gavartin, D. Muñoz Ramo, A. L. Shluger, G. Bersuker, B. H. Lee, Appl. Phys. Lett. 2006, 89, 082908.

[68] P. Deák, B. Aradi, T. Frauenheim, E. Janzén, A. Gali, Phys. Rev. B 2010, 81, 153203.

[69] M. Guidon, J. Hutter, J. VandeVondele, J. Chem. Theory Comput. 2010, 6, 2348.

[70] A. J. Cohen, P. Mori-Sánchez, W. Yang, Chem. Rev. 2012, 112, 289.

[71] J. L. Gavartin, P. V. Sushko, A. L. Shluger, Phys. Rev. B 2003, 67, 035108.

[72] G. Pacchioni, F. Frigoli, D. Ricci, J. A. Weil, Phys. Rev. B 2000, 63, 054102. 
[73] J. Lægsgaard, K. Stokbro, Phys. Rev. Lett. 2001, 86, 2834.

[74] T. Koopmans, Physica 1934, 1, 104.

[75] S. Lany, A. Zunger, Phys. Rev. B 2009, 80, 085202.

[76] T. Stein, L. Kronik, R. Baer, J. Am. Chem. Soc. 2009, 131, 2818.

[77] J. Autschbach, M. Srebro, Acc. Chem. Res. 2014, 47, 2592.

[78] A. Karolewski, L. Kronik, S. Kümmel, J. Chem. Phys. 2013, 138, 204115.

[79] S. Lany, A. Zunger, Phys. Rev. B 2010, 81, 205209.

[80] A. R. Elmaslmane, M. B. Watkins, K. P. McKenna, J. Chem. Theory Comput. 2018, 14, 3740.

[81] A. Mang, K. Reimann, S. Rübenacke, Solid State Commun. 1995, 94, 251.

[82] D. C. Reynolds, D. C. Look, B. Jogai, C. W. Litton, G. Cantwell, W. C. Harsch, Phys. Rev. B 1999, 60, 2340.

[83] A. A. Sokol, S. A. French, S. T. Bromley, C. R. A. Catlow, H. J. J. van Dam, P. Sherwood, Faraday Discuss. 2007, 134, 267.

[84] O. Schirmer, J. Phys. Chem. Solids 1968, 29, 1407.

[85] O. Schirmer, D. Zwingel, Solid State Commun. 1970, 8, 1559.

[86] O. F. Schirmer, Phys. status solidi 2007, 4, 1179.

[87] M. G. Wardle, J. P. Goss, P. R. Briddon, Phys. Rev. B 2005, 71, 155205.

[88] A. Janotti, C. G. Van de Walle, Reports Prog. Phys. 2009, 72, 126501.

[89] Y. Zhukovskii, E. Kotomin, R. Nieminen, A. Stashans, Comput. Mater. Sci. 1997, 7, 285.

[90] J. B. Varley, A. Janotti, C. Franchini, C. G. Van de Walle, Phys. Rev. B 2012, 85, 081109. 
[91] R. J. Bell, P. Dean, Discuss. Faraday Soc. 1970, 50, 55.

[92] T. â. Chang, J. D. Bauer, J. L. Skinner, J. Chem. Phys. 1990, 93, 8973.

[93] J. Dong, D. A. Drabold, Phys. Rev. B 1996, 54, 10284.

[94] D. A. Drabold, U. Stephan, J. Dong, S. M. Nakhmanson, J. Mol. Graph. Model. 1999, 17, 285.

[95] M. Unge, T. Christen, Chem. Phys. Lett. 2014, 613, 15.

[96] J. Strand, M. Kaviani, V. V. Afanas'ev, J. G. Lisoni, A. L. Shluger, Nanotechnology 2018, 29, 125703 .

[97] E. Johlin, L. K. Wagner, T. Buonassisi, J. C. Grossman, Phys. Rev. Lett. 2013, 110, 146805.

[98] J. Tempere, J. T. Devreese, Phys. Rev. B 2001, 64, 104504.

[99] J. Albertsson, S. C. Abrahams, Å. . Kvick, Acta Crystallogr. B Struct. Sci. Cryst. Eng. Mater. 1989, 45, 34 .

[100] A. A. Sokol 2018.

[101] B. K. Meyer, H. Alves, D. M. Hofmann, W. Kriegseis, D. Forster, F. Bertram, J. Christen, A. Hoffmann, M. Straßburg, M. Dworzak, U. Haboeck, A. V. Rodina, Phys. status solidi 2004, 241, 231.

[102] A. Walsh, J. L. F. Da Silva, S.-H. Wei, Chem. Mater. 2009, 21, 5119.

[103] W. Haynes, editor. CRC Handbook of Chemistry and Physics. CRC Press, Florida, 94th edition, 2014.

[104] J. M. Ziman. Static Properties of Solids, 139-144. Cambridge University Press, Cambridge, 2 edition. ISBN 9781139644075, 1972.

[105] A. de Jamblinne de Meux, G. Pourtois, J. Genoe, P. Heremans, J. Phys. D. Appl. Phys. 2015, 48, 435104 . 
[106] J. E. Medvedeva, D. B. Buchholz, R. P. H. Chang, Adv. Electron. Mater. 2017, 3, 1700082.

[107] K. Nomura, H. Ohta, A. Takagi, T. Kamiya, M. Hirano, H. Hosono, Nature 2004, 432, 488.

[108] A. de Jamblinne de Meux, G. Pourtois, J. Genoe, P. Heremans, Phys. Rev. B 2018, 97, 045208.

[109] J. Robertson, J. Non. Cryst. Solids 2008, 354, 2791.

[110] A. de Jamblinne de Meux, G. Pourtois, J. Genoe, P. Heremans, J. Appl. Phys. 2018, 123, 161513.

[111] V. V. Afanas'ev, Adv. Condens. Matter Phys. 2014, 2014, 1.

[112] J. Strand, O. A. Dicks, M. Kaviani, A. L. Shluger, Microelectron. Eng. 2017, 178, 235.

[113] S. Chen, L.-W. Wang, Phys. Rev. B 2014, 89, 014109.

[114] H.-H. Nahm, Y.-S. Kim, D. H. Kim, Phys. status solidi 2012, 249, 1277.

\section{List of Figures}

$1 \quad$ Average radial distribution function from $50 \mathrm{a}-\mathrm{ZnO}$ structures. The steps to generate the amorphous structures is given in Section $\mid 2.1$. Every structure was fully reoptimized by the PBE0-TC-LRC functional and their average and distribution is shown in green and grey. The calculated IP and PBE profiles ${ }^{27}$ are also shown as a comparison. Vertical yellow lines represent the wurtzite bulk $\mathrm{ZnO}$ values. 
2 DoS for neutrally charged a) wurtzite and b) a-ZnO structures using the PBE0TC-LRC functional and a Gaussian smearing of $0.20 \mathrm{eV}$. The top of the valence band (VB) was set to $0.0 \mathrm{eV}$. States in the conduction band (CB) were amplified by a factor of 5 to improve visualization. IPR spectra is shown in red. Higher IPR values denote stronger localization. The a-ZnO DoS and IPR plots were averaged from the 50 different amorphous structures. Inset: CBM amplification of c-ZnO (*) and a-ZnO $(+)$ with Gaussian smearing of 0.20 and $0.001 \mathrm{eV}$. The profiles in the inset were shifted to help comparison. . . . . . . . . . . . . . . . . . . . . . 11

$3 \quad$ The calculated spin density (blue) and atomic structure of the $\mathrm{Li}_{\mathrm{Zn}}$ defect, before and after atomic relaxation. Isosurface is not shown for the unrelaxed and delocalized defect. After relaxation, the hole is localized either axially or nonaxially, as shown in the figure. The direction of the atomic Li-O relaxation is shown by arrows. Energies with respect to the unrelaxed structure are shown below the figures. Green and red

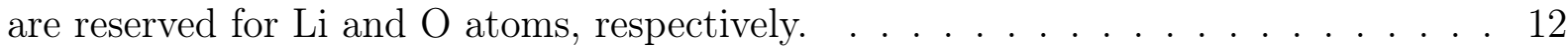

4 The $\mathrm{Zn}-\mathrm{O}-\mathrm{Zn}$ bond angle distribution, from 50 a-ZnO structures, calculated with the PBE0-TC-LRC functional is shown in grey. Average profiles for fully optimized IP, PBE ${ }^{27}$ and PBE0-TC-LRC structures are also displayed. Vertical green lines represent the wurtzite bulk $\mathrm{ZnO}$ values: $\approx 108^{\circ}$ for two $\mathrm{Zn}$ in different hexagonal planes and $\approx 110^{\circ}$ for two Zn in the same hexagonal sheet. . . . . . . . . . . . . . 14

5 Density distribution (with a Gaussian smearing of $\sigma=0.01 \mathrm{~g} \mathrm{~cm}^{-3}$ ), from 50 structures, of the PBE0-TC-LRC a-ZnO structures. IP and DFT distributions $\stackrel{27}{2}$ are also shown for comparison. The wurtzite bulk value at $298 \mathrm{~K}$ is $5.6 \mathrm{~g} \mathrm{~cm}^{-3}$.103] . . . 15

6 The wavefunctions of the HOMO and LUMO of a-ZnO. Grey is reserved for $\mathrm{Zn}$ atoms. Blue and yellow indicate positive and negative isosurfaces..... .18

7 The spin density of the hole polarons (blue) in a-ZnO. a) The hole is centered in an undercoordinate $\mathrm{O}$ ion. b) The hole is centered in a 4-coordinated $\mathrm{Zn} d$ orbital

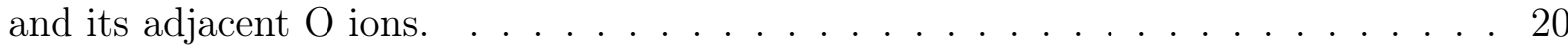


8 Calculated hole trapping energies for 50 structures using $37.5 \%$ and $25 \%$ of the exact HF exchange and 324-atom simulation boxes. The 16 irreversible cases are shown with arrows. The Gaussian smeared charge trapping energy distribution is shown on the right with $\mathrm{a} \sigma=0.05 \mathrm{eV} . \ldots \ldots \ldots \ldots \ldots \ldots$. . . . . . . . . . . 21

$9 \quad$ An example of the the spin density of the most common hole polaron in a-ZnO. a) Before structural optimization, and b) after the full geometry relaxation. . . . . . . 21 